\title{
The General Form of Dual Variables Equations for Elastic Waves in General Elastic Media Layers and Its Applications
}

\author{
S.W. GUO ${ }^{1,} \mathrm{a}^{*}$, J. M. DING ${ }^{2}$ \\ ${ }^{1}$ School of Mechanical Engineering, Southwest Jiaotong University, Chengdu 610031, China \\ ${ }^{2}$ State Key Laboratory of Traction Power, Southwest Jiaotong University, Chengdu 610031, China \\ aguoshiwei@home.swjtu.edu.cn \\ ${ }^{*}$ Corresponding author
}

Keywords: general elastic media, elastic waves in media layers, general form of dual variables equations, eigenvalue problems.

\begin{abstract}
Based on the basic equations of elastic waves in general media, the general form of dual variables equations for plane elastic waves in general elastic media are developed by the transformation of matrix and vector operation. The general form of dual variables equations are generally suitable not only for isotropic and perfect elastic media, but also for anisotropic and viscoelastic media, and adapt to the coupling of various wave types. Putting the elastic coefficients of stiffness matrix of specific medium layer in the general form of dual variables equations, the dual variables equations of the specific medium layer can directly be obtained. The general solving methods for elastic waves problems are presented based on the solutions of dual variables equations. Finally, solving the eigenvalue problems of anisotropic media of cubic system and isotropic media are demonstrated, and the effectiveness and universal applicability of the general form of dual variables equations are indicated.
\end{abstract}

\section{Introduction}

The propagation problems of elastic waves in media layers are the basic and universal problems of continuum mechanics, and are significant in theory and practice of seismic prospecting, structural engineering, nondestructive testing, etc. The propagation problems of elastic waves are also the complex problems because of the various media types, such as perfect elastic medium, viscoelastic medium, isotropic medium and anisotropic medium etc, and of the reflection and transmission problems, general boundary condition problems. However, the analytical and solving methods of these problems have the intrinsic similarity and unity.

For the problems of elastic waves in media layers, the traditional solving methods are based on classical elastic wave equations [1,2]. Elastic wave equations regard displacements as basic variables, and are one kind variable solution methodology classified as Lagrange system theory. Zhong et al. [3-5] presented the dual variables equations of elastic waves. The dual variables equations regard the dual variables of displacement and stress as basic variables, and are two kinds variable solution methodology classified as Hamilton system theory. Compared with wave equations with one kind variable, Hamilton solution system has more advantages. Because dual variables equations regard displacement and stress as basic variables, the stress variables avoid being solved indirectly, and the boundary conditions can be dealt with intuitionisticly and easily. A set of methodology of state-space equations can be applied to solve the dual variables equations of elastic waves, and the properties of elastic wave can be represented intuitively and comprehensively [6-9].

The derivation methods of dual variables equations of elastic waves in references 3-5 are based on energy variation principle and Legendre transform, and they are complicated and nonintuitive. Additionally, they mainly apply to the perfect elastic and isotropic media. Based on the elastic equations of general media, the generalized form of dual variables equations for plane elastic waves in general elastic media layers are deduced in this paper by the transformation operation of matrix form equations. With the transformation operation of matrix and vector, the deducing processes are 
simplified and are easily understandable. The generalized form of dual variables equations are generally suitable not only for isotropic and perfect elastic media, but also for anisotropic and viscoelastic media, and they can adapt to the coupling situation of various wave types.

Dual variables equations are actually the state-space model with a set of methodology that can be directly applied to elastic waves problems. The methodology include variables separation method, eigenfunction expansion method, and transfer matrix method, etc $[10,11]$. The solving methods are unified, well-adapted, and can inherently reveal the relationship among these methods. Based on dual variables equations of elastic waves, this paper introduce the general solving methods for elastic wave problems, and illustrate with the examples of isotropic media and anisotropic media of cubic system.

\section{The General Form of Dual Variables Equations for Elastic Waves}

The multilayers structure of elastic media generally has the parallel plane interfaces, and the media within layers are linear homogeneous media. The rectangular coordinate system is established as shown in Fig. 1. The XOY plane parallel to the media interfaces, and $\mathrm{Z}$ coordinate axis is perpendicular to interfaces.

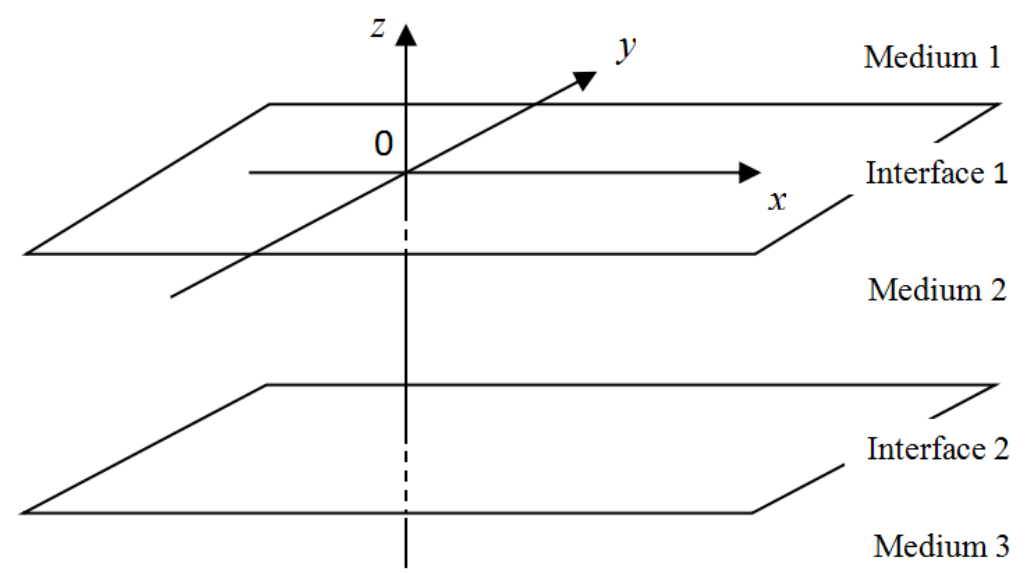

Fig.1 The structure of elastic media layers and the coordinate system

For general solid media, the displacement variables are marked as $u 1=u x, u 2=u y, u 3=u z$, and the stress variables are marked as $\tau 1=\sigma x, \tau 2=\sigma y, \tau 3=\sigma z, \tau 4=\tau y z, \tau 5=\tau z x, \tau 6=\tau x y$. Consequently, the elastic dynamic equations of elastic waves are expressed as matrix form equations given by:

$$
\left[\begin{array}{cccccc}
\frac{\partial}{\partial x} & 0 & 0 & 0 & \frac{\partial}{\partial z} & \frac{\partial}{\partial y} \\
0 & \frac{\partial}{\partial y} & 0 & \frac{\partial}{\partial z} & 0 & \frac{\partial}{\partial x} \\
0 & 0 & \frac{\partial}{\partial z} & \frac{\partial}{\partial y} & \frac{\partial}{\partial x} & 0
\end{array}\right]\left[\begin{array}{c}
\tau_{1} \\
\tau_{2} \\
\tau_{3} \\
\tau_{4} \\
\tau_{5} \\
\tau_{6}
\end{array}\right]=\rho \frac{\partial^{2}}{\partial t^{2}}\left[\begin{array}{c}
u_{1} \\
u_{2} \\
u_{3}
\end{array}\right]-\left[\begin{array}{c}
F_{x} \\
F_{y} \\
F_{z}
\end{array}\right]
$$

Where Fx, Fy, Fz are respectively the $\mathrm{x}, \mathrm{y}, \mathrm{z}$ components of volume force [12]. And from the geometric equations and constitutive equations, another matrix form equations are discribed as:

$$
\left[\begin{array}{llllll}
C_{11} & C_{12} & C_{13} & C_{14} & C_{15} & C_{16} \\
C_{12} & C_{22} & C_{23} & C_{24} & C_{25} & C_{26} \\
C_{13} & C_{23} & C_{33} & C_{34} & C_{35} & C_{36} \\
C_{14} & C_{24} & C_{34} & C_{44} & C_{45} & C_{46} \\
C_{15} & C_{25} & C_{35} & C_{45} & C_{55} & C_{56} \\
C_{16} & C_{26} & C_{36} & C_{46} & C_{56} & C_{66}
\end{array}\right]\left[\begin{array}{lll}
\partial / \partial x & 0 & 0 \\
0 & \partial / \partial y & 0 \\
0 & 0 & \partial / \partial z \\
0 & \partial / \partial z & \partial / \partial y \\
\partial / \partial z & 0 & \partial / \partial x \\
\partial / \partial y & \partial / \partial x & 0
\end{array}\right]\left[\begin{array}{l}
u_{1} \\
u_{2} \\
u_{3}
\end{array}\right]=\left[\begin{array}{l}
\tau_{1} \\
\tau_{2} \\
\tau_{3} \\
\tau_{4} \\
\tau_{5} \\
\tau_{6}
\end{array}\right]
$$

Where the stiffness matrix $\mathrm{C}$ of general media is the symmetric matrix, and the elastic property of medium layer can be entirely described by the stiffness matrix C [13]. 
Supposing the harmonic elastic waves are the plane form waves in XOY plane, the displacement and stress variables are denoted as:

$$
\begin{aligned}
& \boldsymbol{U}=\left[u_{1}(z), u_{2}(z), u_{3}(z)\right]^{T} \exp \left[\left(k_{x} x+k_{y} y-\omega t\right) j\right], \\
& \boldsymbol{\tau}=\left[\tau_{1}(z), \tau_{2}(z), \tau_{3}(z), \tau_{4}(z), \tau_{5}(z), \tau_{6}(z)\right]^{T} \exp \left[\left(k_{x} x+k_{y} y-\omega t\right) j\right]
\end{aligned}
$$

The volume force components are also written as the simple harmonic forms given by:

$$
\left[F_{x}, F_{y}, F_{z}\right]^{T}=\left[f_{x}(z), f_{y}(z), f_{z}(z)\right]^{T} \exp \left[\left(k_{x} x+k_{y} y-\omega t\right) j\right]
$$

For the viscoelastic media, its constitutive relations of stress-strain are also the multiplicative equations as (2) in frequency domain. However, the elements in stiffness matrix $\mathrm{C}$ are the Fourier Transforms of corresponding relaxation functions [14]. So the elements of stiffness matrix $\mathrm{C}$ are the complex numbers, and the wave-number components of $\mathrm{kx}$, ky are also possibly the complex numbers.

Substituting (3), (4) into (1), (2), we have:

$$
\begin{aligned}
& {\left[\begin{array}{llllll}
k_{x} j & 0 & 0 & 0 & \frac{d}{d z} & k_{y} j \\
0 & k_{y} j & 0 & \frac{d}{d z} & 0 & k_{x} j \\
0 & 0 & \frac{d}{d z} & k_{y} j & k_{x} j & 0
\end{array}\right]\left[\begin{array}{l}
\tau_{1}(z) \\
\tau_{2}(z) \\
\tau_{3}(z) \\
\tau_{4}(z) \\
\tau_{5}(z) \\
\tau_{6}(z)
\end{array}\right]=-\rho \omega^{2}\left[\begin{array}{l}
u_{1}(z) \\
u_{2}(z) \\
u_{3}(z)
\end{array}\right]-\left[\begin{array}{l}
f_{x}(z) \\
f_{y}(z) \\
f_{z}(z)
\end{array}\right]} \\
& {\left[\begin{array}{llllll}
C_{11} & C_{12} & C_{13} & C_{14} & C_{15} & C_{16} \\
C_{12} & C_{22} & C_{23} & C_{24} & C_{25} & C_{26} \\
C_{13} & C_{23} & C_{33} & C_{34} & C_{35} & C_{36} \\
C_{14} & C_{24} & C_{34} & C_{44} & C_{45} & C_{46} \\
C_{15} & C_{25} & C_{35} & C_{45} & C_{55} & C_{56} \\
C_{16} & C_{26} & C_{36} & C_{46} & C_{56} & C_{66}
\end{array}\right]\left[\begin{array}{lll}
k_{x} j & 0 & 0 \\
0 & k_{y} j & 0 \\
0 & 0 & d / d z \\
0 & d / d z & k_{y} j \\
d / d z & 0 & k_{x} j \\
k_{y} j & k_{x} j & 0
\end{array}\right]\left[\begin{array}{l}
u_{1}(z) \\
u_{2}(z) \\
u_{3}(z)
\end{array}\right]=\left[\begin{array}{l}
\tau_{1}(z) \\
\tau_{2}(z) \\
\tau_{3}(z) \\
\tau_{4}(z) \\
\tau_{5}(z) \\
\tau_{6}(z)
\end{array}\right]}
\end{aligned}
$$

Merging equations (5) and (6) into one equations group, and adjusting the sequence of some equations and stress components, we obtain:

$$
\left[\begin{array}{lllllllll}
\rho \omega^{2} & 0 & 0 & d / d z & 0 & 0 & k_{x} j & 0 & k_{y} j \\
0 & \rho \omega^{2} & 0 & 0 & d / d z & 0 & 0 & k_{y} j & k_{x} j \\
0 & 0 & \rho \omega^{2} & k_{x} j & k_{y} j & d / d z & 0 & 0 & 0 \\
C_{55} d / d z+a_{11} j & C_{45} d / d z+a_{12} j & C_{35} d / d z+a_{13} j & -1 & 0 & 0 & 0 & 0 & 0 \\
C_{45} d / d z+a_{21} j & C_{44} d / d z+a_{22} j & C_{34} d / d z+a_{23} j & 0 & -1 & 0 & 0 & 0 & 0 \\
C_{35} d / d z+a_{31} j & C_{34} d / d z+a_{32} j & C_{33} d / d z+a_{33} j & 0 & 0 & -1 & 0 & 0 & 0 \\
C_{15} d / d z+a_{41} j & C_{14} d / d z+a_{42} j & C_{13} d / d z+a_{43} j & 0 & 0 & 0 & -1 & 0 & 0 \\
C_{25} d / d z+a_{51} j & C_{24} d / d z+a_{52} j & C_{23} d / d z+a_{53} j & 0 & 0 & 0 & 0 & -1 & 0 \\
C_{56} d / d z+a_{61} j & C_{46} d / d z+a_{62} j & C_{36} d / d z+a_{63} j & 0 & 0 & 0 & 0 & 0 & -1
\end{array}\right]\left[\begin{array}{l}
u_{1}(z) \\
u_{2}(z) \\
u_{3}(z) \\
\tau_{5}(z) \\
\tau_{4}(z) \\
\tau_{3}(z) \\
\tau_{1}(z) \\
\tau_{2}(z) \\
\tau_{6}(z)
\end{array}\right]=-\left[\begin{array}{l}
f_{x}(z) \\
f_{y}(z) \\
f_{z}(z) \\
0 \\
0 \\
0 \\
0 \\
0 \\
0
\end{array}\right]
$$

Where

$a_{11}=C_{15} k_{x}+C_{56} k_{y}, \quad a_{12}=C_{25} k_{y}+C_{56} k_{x}, \quad a_{13}=C_{45} k_{y}+C_{55} k_{x}, \quad a_{21}=C_{14} k_{x}+C_{46} k_{y}, \quad a_{22}=C_{24} k_{y}+C_{46} k_{x}$, $a_{23}=C_{45} k_{x}+C_{44} k_{y}$,

$a_{31}=C_{13} k_{x}+C_{36} k_{y}, \quad a_{32}=C_{23} k_{y}+C_{36} k_{x}, \quad a_{33}=C_{35} k_{x}+C_{34} k_{y}, \quad a_{41}=C_{11} k_{x}+C_{16} k_{y}, \quad a_{42}=C_{16} k_{x}+C_{12} k_{y}$, $a_{43}=C_{15} k_{x}+C_{14} k_{y}$,

$a_{51}=C_{12} k_{x}+C_{26} k_{y}, \quad a_{52}=C_{26} k_{x}+C_{22} k_{y}, \quad a_{53}=C_{25} k_{x}+C_{24} k_{y}, \quad a_{61}=C_{16} k_{x}+C_{66} k_{y}, \quad a_{62}=C_{26} k_{y}+C_{66} k_{x}$, $a_{63}=C_{46} k_{y}+C_{56} k_{x}$.

Deriving from the last three equations of (7), the equations (8) is obtained as:

$$
\left[\begin{array}{l}
\tau_{1}(z) \\
\tau_{2}(z) \\
\tau_{6}(z)
\end{array}\right]=\left[\begin{array}{lll}
C_{15} d / d z+a_{41} j & C_{14} d / d z+a_{42} j & C_{13} d / d z+a_{43} j \\
C_{25} d / d z+a_{51} j & C_{24} d / d z+a_{52} j & C_{23} d / d z+a_{53} j \\
C_{56} d / d z+a_{61} j & C_{46} d / d z+a_{62} j & C_{36} d / d z+a_{63} j
\end{array}\right]\left[\begin{array}{l}
u_{1}(z) \\
u_{2}(z) \\
u_{3}(z)
\end{array}\right]
$$

And deriving from the first six equations of (7), the equations (9) is obtained as: 




Substituting (8) into (9) and arranging it, it yields:

$\left[\begin{array}{llllll}j a_{11} d / d z+\rho \omega^{2}-\left(a_{41} k_{x}+a_{61} k_{y}\right) & j a_{21} d / d z-\left(a_{42} k_{x}+a_{62} k_{y}\right) & j a_{31} d / d z-\left(a_{43} k_{x}+a_{63} k_{y}\right) & d / d z & 0 & 0 \\ j a_{12} d / d z-\left(a_{61} k_{x}+a_{51} k_{y}\right) & j a_{22} d / d z+\rho \omega^{2}-\left(a_{62} k_{x}+a_{52} k_{y}\right) & j a_{32} d / d z-\left(a_{53} k_{y}+a_{63} k_{x}\right) & 0 & d / d z & 0 \\ 0 & 0 & \rho \omega^{2} & k_{x} j & k_{y} j & d / d z \\ C_{55} d / d z+a_{11} j & C_{45} d / d z+a_{12} j & C_{35} d / d z+a_{13} j & -1 & 0 & 0 \\ C_{45} d / d z+a_{21} j & C_{44} d / d z+a_{22} j & C_{34} d / d z+a_{23} j & 0 & -1 & 0 \\ C_{35} d / d z+a_{31} j & C_{34} d / d z+a_{23} j & C_{33} d / d z+a_{33} j & 0 & 0 & -1\end{array}\right]\left[\begin{array}{l}u_{1}(z) \\ u_{2}(z) \\ u_{3}(z) \\ \tau_{5}(z) \\ \tau_{4}(z) \\ \tau_{3}(z)\end{array}\right]=-\left[\begin{array}{l}f_{x}(z) \\ f_{y}(z) \\ f_{z}(z) \\ 0 \\ 0 \\ 0\end{array}\right]$

Isolating the derivative terms of displacement and stress variables from (10), and marking '.' over the variables to denote the derivatives about $\mathrm{z}$, we arrange equation (10) as:

\begin{tabular}{|c|c|c|c|c|c|c|c|c|c|c|c|c|c|c|c|c|c|c|c|c|}
\hline$\dot{u}_{1}(z)$ & {$\left[j a_{11}\right.$} & $j a_{21}$ & $j a_{31}$ & 1 & 0 & $0]^{-1}$ & {$\left[-\rho \omega^{2}+\left(a_{41} k_{x}+a_{61} k_{y}\right)\right.$} & $a_{42} k_{x}+a_{62} k_{y}$ & $a_{43} k_{x}+a_{63} k_{y}$ & 0 & 0 & & $u_{1}(z)$ & {$\left[j a_{11}\right.$} & $j a_{21}$ & $j a_{31}$ & 1 & 0 & 0 & {$\left[f_{x}(z)\right]$} \\
\hline$\dot{u}_{2}(z)$ & $j a_{12}$ & $j a_{22}$ & $j a_{32}$ & 0 & 1 & 0 & $a_{61} k_{x}+a_{51} k_{y}$ & $-\rho \omega^{2}+\left(a_{62} k_{x}+a_{52} k_{y}\right)$ & $a_{63} k_{x}+a_{53} k_{y}$ & 0 & 0 & 0 & $u_{2}(z)$ & $j a_{12}$ & $j a_{22}$ & $j a_{32}$ & 0 & 1 & 0 & $f_{y}(z)$ \\
\hline$\dot{u}_{3}(z)$ & 0 & 0 & 0 & 0 & 0 & 1 & 0 & 0 & $-\rho \omega^{2}$ & $-k_{x} j$ & $-k_{y} j$ & 0 & $u_{3}(z)$ & 0 & 0 & 0 & 0 & 0 & 1 & $f_{z}(z)$ \\
\hline$\dot{\tau}_{5}(z)$ & $C_{55}$ & $C_{45}$ & $C_{35}$ & 0 & 0 & 0 & $-j a_{11}$ & $-j a_{12}$ & $-j a_{13}$ & 1 & $0^{y^{j}}$ & 0 & $\tau_{5}(z)$ & $C_{55}$ & $C_{45}$ & $C_{35}$ & 0 & 0 & 0 & 0 \\
\hline$\dot{\tau}_{4}(z)$ & $C_{45}$ & $C_{44}$ & $C_{34}$ & 0 & 0 & 0 & $-j a_{21}$ & $-j a_{22}$ & $-j a_{23}$ & 0 & 1 & 0 & $\tau_{4}(z)$ & $C_{45}$ & $C_{44}$ & $C_{34}$ & 0 & 0 & 0 & 0 \\
\hline$\left.\dot{\tau}_{3}(z)\right\rfloor$ & $C_{35}$ & $C_{34}$ & $C_{33}$ & 0 & 0 & $0]$ & $-j a_{31}$ & $-j a_{32}$ & $-j a_{33}$ & 0 & 0 & 1 & $\tau_{3}(z)$ & $C_{35}$ & $C_{34}$ & $C_{33}$ & 0 & 0 & $0\rfloor$ & 0 \\
\hline
\end{tabular}

And (11a) can be simplified as:

$\dot{X}(z)=H \cdot X(z)+F(z)$

Equations (11) is the generalized form of dual variables equations for plane elastic waves in general elastic media layers, where the displacement components $\mathrm{u} 1, \mathrm{u} 2$, $\mathrm{u} 3$ and stress components $\tau 5, \tau 4, \tau 3$ are the basic dual variables. Equations (11) are first order ordinary differential equations of dual variables about $\mathrm{z}$, namely the state space equations form, which can be considered as the transmission line model of elastic waves in media layers. For the propagation problems of elastic waves, the volume force components can be neglected, and the second item about volume force in the right side of equations (11) can be removed. For the stiffness matrix $\mathrm{C}$ of general media, the corresponding matrix $\mathrm{H}$ is Hamiltonian matrix, then equations (11) are also called Hamiltonian dual equations.

The above deduction of the generalized form of dual variables equations (11) are based on the basic elastic equations, thus equations (11) are generally suitable not only for isotropic and perfect elastic media, but also anisotropic and viscoelastic media, and can adapt to the general coupling situation of various wave types. The elastic properties of medium layer are entirely described by the stiffness matrix C. Putting related constants of wave numbers and the elastic coefficients of stiffness matrix $C$ of specific medium layer into equations (11), we can directly get the dual variables equations of the specific medium layer.

Although the general form of dual variables equations (11) look complicated, the inverse matrix in equations (11) can be easily solved by inverse matrix formula of block matrix. And equations (11) can be greatly simplified in practical calculations because some elements in stiffness matrix $\mathrm{C}$ will become zeros, and the number of independent coefficients of stiffness matrix $\mathrm{C}$ will reduce when the degree of symmetry of medium property about coordinate axis and plane increase [15]. Additionally, parts of dual variables can be decoupled, namely various type of elastic waves can be decomposed when elastic waves propagate along the special direction in media layer [16]. Thus, we can get the independent lower order dual variables equations whose analysis and solution are greatly simplified.

Other physical variables of elastic waves problems can generally be expressed as algebraic equations of dual variables. For example, based on equations (8), the other three stress components $\tau 1, \tau 2, \tau 6$ can be expressed as: 


$$
\left[\begin{array}{c}
\tau_{1}(z) \\
\tau_{2}(z) \\
\tau_{6}(z)
\end{array}\right]=\left[\begin{array}{lll}
C_{15} & C_{14} & C_{13} \\
C_{25} & C_{24} & C_{23} \\
C_{56} & C_{46} & C_{36}
\end{array}\right]\left[\begin{array}{l}
\dot{u}_{1}(z) \\
\dot{u}_{2}(z) \\
\dot{u}_{3}(z)
\end{array}\right]+\left[\begin{array}{ccc}
a_{41} j & a_{42} j & a_{43} j \\
a_{51} j & a_{52} j & a_{53} j \\
a_{61} j & a_{62} j & a_{63} j
\end{array}\right]\left[\begin{array}{l}
u_{1}(z) \\
u_{2}(z) \\
u_{3}(z)
\end{array}\right]
$$

Where $\left[\dot{u}_{1}(z), \dot{u}_{2}(z), \dot{u}_{3}(z)\right]^{T}$ can be directly expressed as the algebraic expression of dual variables from the first three equations of (11). Substituting the expression into (12a) and arranging it, we can get the algebraic expression of stress components $\tau 1, \tau 2$, $\tau 6$ with dual variables:

$$
Y(z)=B^{\bullet} X(z)
$$

Where $\mathrm{Y}(\mathrm{z})=[\tau 1(\mathrm{z}), \tau 2(\mathrm{z}), \tau 6(\mathrm{z})] \mathrm{T}$. Equation (12) can be called output equations whose solutions can be directly gotten with the solutions of dual variables equations (11).

Combining equations (11) and (12), we can get the state-space model of elastic waves in general media layers with a set of methodology, whose theories and methods can be intuitively applied in elastic waves problems.

\section{The General Solving Methods for Elastic Waves Problems in Elastic Layer}

The dual variables equations of media layers are significant foundations for solving the general propagation problems and dynamic response problems of elastic waves in multilayer media. For specific elastic medium layer, we can directly obtain its state-space model of elastic waves by putting its elastic coefficients of stiffness matrix into equations (11), (12). Then we can apply the methodology of state-space model to solve the elastic wave problems. The general solving methods for elastic waves problems can be described as follows:

\section{The Dual Variables Equations of Media Layers}

When a plane elastic wave is incident on elastic multilayers structure, the wave-number components of $\mathrm{X}$, Y direction, namely $\mathrm{kx}$ and $\mathrm{ky}$ are the definite constants. According to general Snell Law [17], the wave-number components of $\mathrm{X}, \mathrm{Y}$ in every layer are all equal to $\mathrm{kx}$, ky. Substituting kx, ky and every layer's stiffness matrix into equation (11), we can obtain the dual variables equations of every layer.

\section{The Eigenvalue Problems of Dual Variables Equation of Medium Layer}

Based on eigenvalue problems of dual variables equation of medium layer, we can get the eigenvalues, namely the wave-number components of $\mathrm{Z}$ direction $\mathrm{kz}$, and the corresponding eigenvectors, namely the relative values of dual variables of corresponding wave components. With the variables separation methods and eigenfunctions expansion methods, we can get the superposition form solutions of dual variables in every layer. The superposition principle can be applied in solving reflection-transmission problems on layer interfaces, from which Zoeppritz equations to solve the reflection-transmission coefficients can be derived $[18,19]$.

\section{Transfer Matrix Method of Dual Variables for Elastic Waves in Media Layers}

Solving the state transition matrix of dual variables equations, we can get the transfer form solutions of dual variables of elastic waves in media layers, and moreover obtain the transfer matrix method of dual variables for elastic waves in media layers. The transfer matrix method intuitively expresses the relationship of dual variables between interfaces of media layer [20,21], and is suitable to solve the boundary conditions problems of multilayers structure and the elastic wave impedance problems of media layers.

Regarding the displacement variables and corresponding stress variables as basic variables, dual variables equations can avoid the indirect solution of stress variables, and are conducive to dealing with the boundary conditions, and can express the properties of elastic waves intuitively and comprehensively. The solving methods of elastic waves problems based on dual variables equations are unified, well-adapted, and can easily reveal the relationships among them.

The examples of anisotropic media of cubic system and isotropic media are illustrated to show the eigen-solutions of dual variables equations as below. 


\section{The Analysis of Anisotropic Media of Cubic System}

The generalized form of dual variables equations (11) is suitable for various types of anisotropic media. For anisotropic media of cubic system, when the coordinate system consistent with the cubic crystal axes $\mathrm{X}, \mathrm{Y}, \mathrm{Z}$, its stiffness matrix is:

$$
C=\left[\begin{array}{llllll}
c_{11} & c_{12} & c_{12} & 0 & 0 & 0 \\
c_{12} & c_{11} & c_{12} & 0 & 0 & 0 \\
c_{12} & c_{12} & c_{11} & 0 & 0 & 0 \\
0 & 0 & 0 & c_{44} & 0 & 0 \\
0 & 0 & 0 & 0 & c_{44} & 0 \\
0 & 0 & 0 & 0 & 0 & c_{44}
\end{array}\right]
$$

Where includes three independent elastic coefficients. Substituting the elements of stiffness matrix $\mathrm{C}$ into equations (11) (and ignoring the volume force components), we can get the dual variables equations of plane elastic waves in cubic crystal layer as:

$$
\left[\begin{array}{l}
\dot{u}_{1}(z) \\
\dot{u}_{2}(z) \\
\dot{u}_{3}(z) \\
\dot{\tau}_{5}(z) \\
\dot{\tau}_{4}(z) \\
\dot{t}_{3}(z)
\end{array}\right]=\left[\begin{array}{llllll}
0 & 0 & -j k_{x} & 1 / c_{44} & 0 & 0 \\
0 & 0 & -j k_{y} & 0 & 1 / c_{44} & 0 \\
-j c_{12} k_{x} / c_{11} & -j c_{12} k_{y} / c_{11} & 0 & 0 & 0 & 1 / c_{11} \\
-\rho \omega^{2}+c_{11} k_{x}{ }^{2}+c_{44} k_{y}{ }^{2}-c_{12}{ }^{2} k_{x}{ }^{2} / c_{11} & \left(c_{12} c_{11}+c_{44} c_{11}-c_{12}{ }^{2}\right) k_{x} k_{y} / c_{11} & 0 & 0 & 0 & -j c_{12} k_{x} / c_{11} \\
\left(c_{12} c_{11}+c_{44} c_{11}-c_{12}{ }^{2}\right) k_{x} k_{y} / c_{11} & -\rho \omega^{2}+\left(c_{11}^{2} k_{y}{ }^{2}+c_{44} c_{11} k_{x}{ }^{2}-c_{12}{ }^{2} k_{y}{ }^{2}\right) / c_{11} & 0 & 0 & 0 & -j c_{12} k_{y} / c_{11} \\
0 & 0 & -\rho \omega^{2} & -j k_{x} & -j k_{y} & 0
\end{array}\right]\left[\begin{array}{l}
u_{1}(z) \\
u_{2}(z) \\
u_{3}(z) \\
\tau_{5}(z) \\
\tau_{4}(z) \\
\tau_{3}(z)
\end{array}\right]
$$

And when plane waves propagate in coordinate plane $\mathrm{XOZ}$, then $\mathrm{ky}=0$. And substituting $\mathrm{ky}=0$ into equation (14), we can get the dual variables equations of two-dimensional plane waves as:

$$
\left[\begin{array}{l}
\dot{u}_{1}(z) \\
\dot{u}_{2}(z) \\
\dot{u}_{3}(z) \\
\dot{\tau}_{5}(z) \\
\dot{\tau}_{4}(z) \\
\dot{\tau}_{3}(z)
\end{array}\right]=\left[\begin{array}{llllll}
0 & 0 & -j k_{x} & 1 / c_{44} & 0 & 0 \\
0 & 0 & 0 & 0 & 1 / c_{44} & 0 \\
-j c_{12} k_{x} / c_{11} & 0 & 0 & 0 & 0 & 1 / c_{11} \\
-\rho \omega^{2}+\left(c_{11}^{2}-c_{12}{ }^{2}\right) k_{x}{ }^{2} / c_{11} & 0 & 0 & 0 & 0 & -j c_{12} k_{x} / c_{11} \\
0 & -\rho \omega^{2}+c_{44} k_{x}^{2} & 0 & 0 & 0 & 0 \\
0 & 0 & -\rho \omega^{2} & -j k_{x} & 0 & 0
\end{array}\right]\left[\begin{array}{l}
u_{1}(z) \\
u_{2}(z) \\
u_{3}(z) \\
\tau_{5}(z) \\
\tau_{4}(z) \\
\tau_{3}(z)
\end{array}\right]
$$

Rearranging the displacement and stress components in equation (15), we can obtain two decoupled dual variables equations which indicate two typies of elastic waves:

$$
\begin{aligned}
& {\left[\begin{array}{l}
\dot{u}_{1}(z) \\
\dot{u}_{3}(z) \\
\dot{\tau}_{5}(z) \\
\dot{\tau}_{3}(z)
\end{array}\right]=\left[\begin{array}{llll}
0 & -j k_{x} & 1 / c_{44} & 0 \\
-j c_{12} k_{x} / c_{11} & 0 & 0 & 1 / c_{11} \\
-\rho \omega^{2}+\left(c_{11}^{2}-c_{12}{ }^{2}\right) k_{x}{ }^{2} / c_{11} & 0 & 0 & -j c_{12} k_{x} / c_{11} \\
0 & -\rho \omega^{2} & -j k_{x} & 0
\end{array}\right]\left[\begin{array}{l}
u_{1}(z) \\
u_{3}(z) \\
\tau_{5}(z) \\
\tau_{3}(z)
\end{array}\right]} \\
& {\left[\begin{array}{l}
\dot{u}_{2}(z) \\
\dot{\tau}_{4}(z)
\end{array}\right]=\left[\begin{array}{ll}
0 & 1 / c_{44} \\
-\rho \omega^{2}+c_{44} k_{x}^{2} & 0
\end{array}\right]\left[\begin{array}{l}
u_{2}(z) \\
\tau_{4}(z)
\end{array}\right]}
\end{aligned}
$$

For equations (16), supposing its dual variables are the form of:

$$
\left[u_{1}(z), u_{3}(z), \tau_{5}(z), \tau_{3}(z)\right]^{T}=\left[U_{1}, U_{3}, T_{5}, T_{3}\right]^{T} e^{\eta z}
$$

And substituting (18) into (16) and solving its eigenvalue problems, we can obtain the eigenvalues $\eta 1,2= \pm \mathrm{kzPj}, \eta 3,4= \pm \mathrm{kzS} 2 \mathrm{j}$, where kzP, kzS2 are respectively:

$$
k_{z P}, k_{z S 2}=\mathrm{PV}\left(\frac{-P \pm\left(P^{2}-4 c_{11} c_{44}\left(c_{11} k_{x}^{2}-\rho \omega^{2}\right)\left(c_{44} k_{x}^{2}-\rho \omega^{2}\right)\right)^{1 / 2}}{2 c_{11} c_{44}}\right)^{1 / 2}
$$

Where $\mathrm{P}=(\mathrm{c} 112-\mathrm{c} 122-2 \mathrm{c} 12 \mathrm{c} 44) \mathrm{kx} 2-(\mathrm{c} 11+\mathrm{c} 44) \rho \omega 2$, and $\mathrm{PV}(\mathrm{z}) 1 / 2$ indicates the main value of square root of complex $z$. The eigenvector corresponding with $k z j(= \pm k z P j, \pm k z S 2 j)$ is: 


$$
\left[\begin{array}{l}
1 \\
\frac{-k_{z} k_{x}\left(k_{z}^{2} c_{11} c_{44}+k_{x}^{2}\left(c_{11}^{2}-c_{12}^{2}-c_{12} c_{44}\right)-\rho \omega^{2}\left(c_{44}+c_{12}+c_{11}\right)\right)}{\left(c_{44} k_{x}^{2}-\rho \omega^{2}\right)\left(k_{z}^{2} c_{11}-k_{x}^{2} c_{12}-\rho \omega^{2}\right)} \\
\frac{j k_{z} c_{44}\left(\left(\rho \omega^{2}\right)^{2}+\rho \omega^{2}\left(-k_{z}^{2} c_{11}+2 c_{12} k_{x}^{2}+c_{11} k_{x}^{2}\right)+k_{x}^{4}\left(c_{12}^{2}-c_{11}^{2}\right)\right)}{\left(c_{44} k_{x}^{2}-\rho \omega^{2}\right)\left(k_{z}^{2} c_{11}-k_{x}^{2} c_{12}-\rho \omega^{2}\right)} \\
\frac{-j k_{x}\left(\rho \omega^{2}\left(c_{12}+c_{11}\right)+k_{x}^{2}\left(c_{12}^{2}-c_{11}^{2}\right)\right)}{\left(k_{z}^{2} c_{11}-k_{x}^{2} c_{12}-\rho \omega^{2}\right)}
\end{array}\right]
$$

The eigenvector (20) is the dual variables of corresponding wave components normalized by $\mathrm{u} 1(\mathrm{z})$. The results of eigenvalue problem above correspond with the characteristics of Hamilton matrix, that is, the eigenvalues are opposite numbers in pairs which indicate the opposite directions that elastic waves components propagate or decay along. Based on the eigenvector (20), every wave components' polarization direction of particle vibration and the wave speed in different propagation direction can be computed out. And it can be verified that the polarization directions of particle vibration are neither perpendicular nor parallel to the propagation direction. Thus, (20) indicates the coupled quasi-longitudinal wave and quasi-transverse wave, and (16) are the dual variables equations of quasi-longitudinal wave and quasi-transverse wave, kzP and kzS2 are respectively the $\mathrm{Z}$ components of wave-numbers of quasi-longitudinal wave and quasi-transverse wave in anisotropic media of cubic system. From the above analysis, it is demonstrated that the concepts and characteristics of propagation problems of elastic waves can be derived from the eigenvalue problems of dual variables equations.

Similarly, for equations (17), supposing its dual variables form as $[\mathrm{u} 2(\mathrm{z}), \tau 4(\mathrm{z})] \mathrm{T}=[\mathrm{U} 2, \mathrm{~T} 4] \mathrm{Te} z \mathrm{z}$, and substituting it into (17), we can solve the eigenvalues as $\eta 1,2= \pm \mathrm{kzS} 1 \mathrm{j}$, where $\mathrm{kzS} 1$ is the $\mathrm{Z}$ component of wave-number which meet the relation of $\mathrm{kzS} 12+\mathrm{kx} 2=\rho \omega 2 / \mathrm{c} 44=\mathrm{kS} 2$. The eigenvector $[1, \mathrm{c} 44 \mathrm{kz}] \mathrm{T}$ corresponding to eigenvalue $\mathrm{kzj}(= \pm \mathrm{kzS} 1 \mathrm{j})$ is the dual variables of corresponding wave components $[\mathrm{u} 2(\mathrm{z}), \tau 4(\mathrm{z})] \mathrm{T}$ normalized by $\mathrm{u} 2(\mathrm{z})$. From the eigenvector, we learn that the propagation direction is perpendicular to the polarization direction of vibration particle in elastic wave components. So, it's the pure shear wave with Y polarization, and (17) is the dual variables equations of pure shear wave in cubic crystal media. The results above are equivalent to that of Christoffel equations [22], and can reveal the relation among stress and displacement variables of wave components with more intuitive and clear methods.

\section{The Analysis of Isotropic Media}

For isotropic media, the stiffness matrix of constitutive equation is written as:

$$
C=\left[\begin{array}{llllll}
\lambda+2 \mu & \lambda & \lambda & 0 & 0 & 0 \\
\lambda & \lambda+2 \mu & \lambda & 0 & 0 & 0 \\
\lambda & \lambda & \lambda+2 \mu & 0 & 0 & 0 \\
0 & 0 & 0 & \mu & 0 & 0 \\
0 & 0 & 0 & 0 & \mu & 0 \\
0 & 0 & 0 & 0 & 0 & \mu
\end{array}\right]
$$

Where there are two independent elastic coefficents $\lambda$ and $\mu$. Substituting elements of (21) into equation (11), we can get the dual variables equation of plane elastic wave in isotropic media layer:

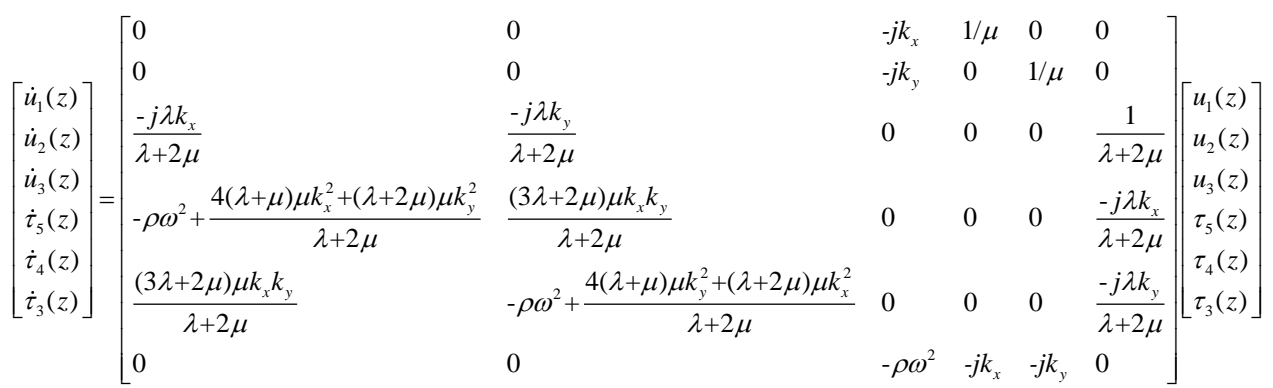


When plane waves propagate in coordinate plane $\mathrm{XOZ}$, then $\mathrm{ky}=0$. Substituting $\mathrm{ky}=0$ into equation (22), we can get the dual variables equations of two-dimensional plane waves as:

$$
\left[\begin{array}{l}
\dot{u}_{1}(z) \\
\dot{u}_{2}(z) \\
\dot{u}_{3}(z) \\
\dot{\tau}_{5}(z) \\
\dot{\tau}_{4}(z) \\
\dot{\tau}_{3}(z)
\end{array}\right]=\left[\begin{array}{llllll}
0 & 0 & -j k_{x} & 1 / \mu & 0 & 0 \\
0 & 0 & 0 & 0 & 1 / \mu & 0 \\
\frac{-j \lambda k_{x}}{\lambda+2 \mu} & 0 & 0 & 0 & 0 & \frac{1}{\lambda+2 \mu} \\
-\rho \omega^{2}+\frac{4(\lambda+\mu) \mu k_{x}^{2}}{\lambda+2 \mu} & 0 & 0 & 0 & 0 & \frac{-j \lambda k_{x}}{\lambda+2 \mu} \\
0 & -\rho \omega^{2}+\mu k_{x}^{2} & 0 & 0 & 0 & 0 \\
0 & 0 & -\rho \omega^{2} & -j k_{x} & 0 & 0
\end{array}\right]\left[\begin{array}{l}
u_{1}(z) \\
u_{2}(z) \\
u_{3}(z) \\
\tau_{5}(z) \\
\tau_{4}(z) \\
\tau_{3}(z)
\end{array}\right]
$$

Rearranging the displacement and stress components in equation (23), we obtain two decoupled dual variables equations:

$$
\begin{aligned}
& {\left[\begin{array}{l}
\dot{u}_{2}(z) \\
\dot{\tau}_{4}(z)
\end{array}\right]=\left[\begin{array}{ll}
0 & 1 / \mu \\
-\rho \omega^{2}+\mu k_{x}^{2} & 0
\end{array}\right]\left[\begin{array}{l}
u_{2}(z) \\
\tau_{4}(z)
\end{array}\right]} \\
& {\left[\begin{array}{l}
\dot{u}_{1}(z) \\
\dot{u}_{3}(z) \\
\dot{\tau}_{5}(z) \\
\dot{\tau}_{3}(z)
\end{array}\right]=\left[\begin{array}{llll}
0 & -j k_{x} & 1 / \mu & 0 \\
\frac{-j \lambda k_{x}}{\lambda+2 \mu} & 0 & 0 & \frac{1}{\lambda+2 \mu} \\
-\rho \omega^{2}+\frac{4(\lambda+\mu) \mu k_{x}^{2}}{\lambda+2 \mu} & 0 & 0 & \frac{-j \lambda k_{x}}{\lambda+2 \mu} \\
0 & -\rho \omega^{2} & -j k_{x} & 0
\end{array}\right]\left[\begin{array}{l}
u_{1}(z) \\
u_{3}(z) \\
\tau_{5}(z) \\
\tau_{3}(z)
\end{array}\right]}
\end{aligned}
$$

Where (24) is the dual variables equations of SH wave, and (25) is the dual variables equations of P-SV wave in isotropic media. The results are similar to that of reference [3].

We just discuss the eigenvalue problems of equation (17) as follows. Supposing its dual variables are form of:

$\left[u_{1}(z), u_{3}(z), \tau_{5}(z), \tau_{3}(z)\right]^{T}=\left[U_{1}, U_{3}, T_{5}, T_{3}\right]^{T} e^{\eta z}$

And substituting (26) into (25), we can solve the eigenvalues as:

$\eta 1,2= \pm \mathrm{kzPj}, \eta 3,4= \pm \mathrm{kzSj}$

Where $\mathrm{kzP}=\mathrm{PV}(\mathrm{kP} 2-\mathrm{kx} 2) 1 / 2$ is the $\mathrm{Z}$ component of wave-numbers of $\mathrm{P}$-wave, $\mathrm{kP}=\omega / \mathrm{cP}$ is the wave-numbers of $\mathrm{P}$-wave, and $c_{P}=\sqrt{(\lambda+2 \mu) / \rho}$ is the wave speed of $\mathrm{P}$-wave; $\mathrm{kzS}=\mathrm{PV}(\mathrm{kS} 2-\mathrm{kx} 2) 1 / 2$ is the $\mathrm{Z}$ component of wave-numbers of $\mathrm{S}$-wave, $\mathrm{kS}=\omega / \mathrm{cS}$ is the wave-numbers of $\mathrm{S}$-wave, and $c_{S}=\sqrt{\mu / \rho}$ is the wave speed of $\mathrm{S}$-wave.

The eigenvectors corresponding with eigenvalues (27) are solved as:

$$
\psi_{1}=\left[\begin{array}{l}
1 \\
k_{z P} / k_{x} \\
2 \mu k_{z P} j \\
j \mu\left(k_{z S}^{2}-k_{x}^{2}\right) / k_{x}
\end{array}\right], \quad \psi_{2}=\left[\begin{array}{l}
1 \\
-k_{z P} / k_{x} \\
-2 \mu k_{z P} j \\
j \mu\left(k_{z S}^{2}-k_{x}^{2}\right) / k_{x}
\end{array}\right], \quad \psi_{3}=\left[\begin{array}{l}
1 \\
-k_{x} / k_{z S} \\
j \mu\left(k_{z S}^{2}-k_{x}^{2}\right) / k_{z S} \\
-j 2 \mu k_{x}
\end{array}\right] \quad \psi_{4}=\left[\begin{array}{l}
1 \\
k_{x} / k_{z S} \\
-j \mu\left(k_{z S}^{2}-k_{x}^{2}\right) / k_{z S} \\
-j 2 \mu k_{x}
\end{array}\right]
$$

Where $\Psi 1,2$ are the dual variables of $\mathrm{P}$-wave components corresponding with $\pm \mathrm{kzP}$, and $\Psi 3,4$ are the dual variables of $\mathrm{SV}$-wave components corresponding with $\pm \mathrm{kzS}$. The vectors of (28) are normalized by $\mathrm{u} 1(\mathrm{z})$, and they can also be normalized by particle displacement amplitude as follow when wave components are uniform plane waves:

$$
\psi_{1}=\left[\begin{array}{l}
\sin \theta_{P} \\
\cos \theta_{P} \\
j \mu k_{P} \sin 2 \theta_{P} \\
j \mu k_{S}^{2} / k_{P} \cdot \cos 2 \theta_{S}
\end{array}\right], \quad \psi_{2}=\left[\begin{array}{l}
\sin \theta_{P} \\
-\cos \theta_{P} \\
-j \mu k_{P} \sin 2 \theta_{P} \\
j \mu k_{S}^{2} / k_{P} \cdot \cos 2 \theta_{S}
\end{array}\right], \quad \psi_{3}=\left[\begin{array}{l}
\cos \theta_{S} \\
-\sin \theta_{S} \\
j \mu k_{S} \cos 2 \theta_{S} \\
-j \mu k_{S} \sin 2 \theta_{S}
\end{array}\right], \quad \psi_{4}=\left[\begin{array}{l}
\cos \theta_{S} \\
\sin \theta_{S} \\
-j \mu k_{S} \cos 2 \theta_{S} \\
-j \mu k_{S} \sin 2 \theta_{S}
\end{array}\right]
$$


Where $\theta \mathrm{P}=\operatorname{tg}-1(\mathrm{kx} / \mathrm{kzP}), \theta \mathrm{S}=\operatorname{tg}-1(\mathrm{kx} / \mathrm{kzS})$ indicate respectively the propagation direction of P-wave, SV-wave components. So the dual variables of P-SV uniform plane waves can be expressed as the superposition solution form as:

$$
\left[\begin{array}{l}
u_{1}(z) \\
u_{3}(z) \\
\tau_{5}(z) \\
\tau_{3}(z)
\end{array}\right]\left[\begin{array}{llll}
\sin \theta_{P} & \sin \theta_{P} & \cos \theta_{S} & \cos \theta_{S} \\
\cos \theta_{P} & -\cos \theta_{P} & -\sin \theta_{S} & \sin \theta_{S} \\
j \mu k_{P} \sin 2 \theta_{P} & -j \mu k_{P} \sin 2 \theta_{P} & j \mu k_{S} \cos 2 \theta_{S} & -j \mu k_{S} \cos 2 \theta_{S} \\
j \mu k_{S}^{2} / k_{P} \cdot \cos 2 \theta_{S} & j \mu k_{S}^{2} / k_{P} \cdot \cos 2 \theta_{S} & -j \mu k_{S} \sin 2 \theta_{S} & -j \mu k_{S} \sin 2 \theta_{S}
\end{array}\right]\left[\begin{array}{cccc}
e^{j k_{k} z z} & 0 & 0 & 0 \\
0 & e^{-j k_{s p} z} & 0 & 0 \\
0 & 0 & e^{j k_{S S} z} & 0 \\
0 & 0 & 0 & e^{-j k_{k_{S} z} z}
\end{array}\right]\left[\begin{array}{c}
A_{1} \\
A_{2} \\
B_{1} \\
B_{2}
\end{array}\right]
$$

Where A1, A2 are respectively the displacement amplitudes of $\mathrm{P}$-wave components propagating along $\pm \mathrm{z}$ directions, and $\mathrm{B} 1, \mathrm{~B} 2$ are respectively the displacement amplitudes of $\mathrm{SV}$-wave components propagating along $\pm \mathrm{z}$ directions. (30) can be regarded as the analytical basis for reflection and refraction problems of P-SV uniform plane waves in isotropic media. Combining with the boundary conditions of multilayers structure, we can intuitively deduce Zoeppritz equations for solving the reflection and refraction coefficients of P-SV waves [23,24].

\section{Conclusions}

The dual variables equations of elastic media are significant foundations for solving the propagation problems and dynamic response problems for elastic waves in multilayer structure. Based on basic equations of elastic waves in general elastic media, this paper develop the generalized form of dual variables equations for plane elastic waves in general media layers by the transformation operation of matrix and vector. To sum up the above discussion, the conclusions are given as following:

(1) Comparing with the deducing methods based on energy variation principle and Legendre transform, the transformation operations of matrix form equations are intuitive and simple. And the generalized form of dual variables equations are generally suitable not only for isotropic and perfect elastic media, but also for anisotropic and viscoelastic media, and can adapt to the coupling situation of various wave types.

(2) For specific media layer, we can directly get its dual variables equations of elastic waves by putting the elastic coefficients of stiffness matrix into the generalized form of dual variables equations. And from dual variables equations of elastic waves, we can analyze and solve the elastic wave problems. That is, we can get the superposition form solutions of dual variables of wave components from solving the eigenvalue problems of dual variables equations, and we can get the transfer form solutions of dual variables of elastic waves from solving the state transition matrix of dual variables equation, and so on. The solving methods based on dual variables equations are unified, intuitive, and well-adapted.

(3) The examples of anisotropic media of cubic system and isotropic media are illustrated to get their dual variables equations from the generalized form of dual variables equations. By solving the eigenvalue problems of dual variables equation we can achieve the decoupling of various wave components, solve the wave-numbers of various wave components, and get the superposition form solutions of dual variables for elastic waves, etc. And it is demonstrated that the results are identical with the conclusion of reference literature, thus indicate the effectiveness and universal applicability of generalized form of dual variables equations in elastic wave problems of media layers.

\section{Acknowledgments}

This work was supported by the National Natural Science Foundation of China (51305358), the Fundamental Research Funds for the Central Universities (2682014CX014EM) and Scientific Research Supporting Project for High-level Personnel of Emei Campus, Southwest Jiaotong University (10201X10096015). 


\section{References}

[1] J. D. Achenbach, Wave Propagation in Elastic Solids. (North-Holland, 2012).

[2] J. Miklowitz, The theory of elastic waves and waveguides. (North-Holland, 2012).

[3] W. X. Zhong, Symplectic Solution Methodology in Applied Mechanics. (Higher Education Press, 2006).

[4] W. X. Zhong, Duality System in Applied Mechanics and Optimal Control. (Kluwer Academic Publishers, 2004).

[5] W. X. Zhong, A New Systematic Methodology of Elasticity. (Dalian University of Technology Press, 1995).

[6] Y. H. Zhang and Y. B. Ma, A wave propagation method in symplectic space for vibration analysis of thin plates, Journal of Vibration and Shock, 33, 1 (2014).

[7] S. Y. Bao and Z. C. Deng, A type of model and ordinary solutions for static bending and free vibration problems of moderate thick plate in Hamilton system, Journal of dynamics and control, $10,121(2012)$

[8] R. Li, Hamiltonian solution approach for the problems of rectangular plates, $\mathrm{PhD}$ thesis, Dalian University of Technology, (LN, China, 2012).

[9] H. Z. Jia, A Method of Hamiltonian System for Reinforcement of Cracked Structures, PhD thesis, Dalian University of Technology, (LN,China,2012).

[10]W. X. Zhong and Z. G. Wu, Some Issues in Theory and Computation of State-space Control: An Analytical Structural Mechanics Viewpoint, Aerospace Control, 25, 3(2007).

[11]Q. Gao, Wave Propagation, Robust Control and Symplectic Method in Hamilton Systems, PhD thesis, Dalian University of Technology, (LN,China,2006).

[12]L. M. Brekhovskikh, Waves in Layered Media, 2nd edn. (Academic Press, 2012).

[13]J. M. Carcione, Wave Fields in Real Media: Wave Propagation in Anisotropic, Anelastic, Porous and Electromagnetic Media, 3rd edn. (Eslevier, 2015).

[14]E. S. Krebes, The Viscoelastic Reflection/Transmission Problem: Two special cases, the Seismological Society of America, 73, 1673 (1984).

[15]F. I. Fedorov, Theory of Elastic Waves in Crystals, (Springer Science \& Business Media, 2013).

[16]L. M. Brekhovskikh and O. A. Godin, Acoustics of Layered Media I: Plane and Quasi-Plane Waves, (Springer-Verlag, 2012).

[17]M. A. Slawinski and R. A. Slawinski, A generalized form of Snell's law in anisotropic media, Geophysics, 65, 632 (2000).

[18]W. X. Zhong and F. W. Williams, The eigensolutions of wave propagation for repetitive structures, Structural engineering and mechanics, l, 47 (1993).

[19]J. M. Renno and B. R. Mace, Calculation of reflection and transmission coefficients of joints using a hybrid finite element / wave and finite element approach, Journal of Sound and Vibration,332, 2149 (2013).

[20]W. X. Zhong and J. H. Lin, Computational structural mechanics and optimal control-the simulation of substructural chain theory to linear quadratic optimal control problems, International Journal for Numerical Methods in Engineering, 33, 197 (1992).

[21]B. L. N. Kennett, Seismic wave propagation in stratified media, (ANU E Press, 2009). 
[22]B. A. Auld, Acoustic fields and waves in solids (John Weley \&Sons, 1973).

[23]W. X. Zhong, the method of separation of variables and Hamilton system, Chinese Journal of Computational Mechanics, 8, 229 (1991).

[24]Y. H. Wang, Approximations to the Zoeppritz equations and their use in AVO analysis, Geophysics, 64, 1920 (1999). 